Annales Geophysicae (2002) 20: 329-340 (c) European Geophysical Society 2002

\title{
Statistical study of transient plasma structures in magnetotail lobes and plasma sheet boundary layer: Interball-1 observations
}

\author{
E. E. Grigorenko ${ }^{1,2}$, A. Fedorov ${ }^{3,}$, and L. M. Zelenyi ${ }^{2}$ \\ ${ }^{1}$ Skobeltzyn Institute of Nuclear Physics of Moscow State University, Vorobjevy Gory, 117234, Moscow, Russia \\ ${ }^{2}$ Space Research Institute, 84/32, Profsojuznaja, 117810, Moscow, Russia \\ ${ }^{3}$ CESR - CNRS, 9, av. de Colonel-Roche, 31400, Toulouse, France \\ *on leave from Space Research Institute, Moscow
}

Received: 31 January 2001 - Revised: 24 September 2001 - Accepted: 21 September 2001

\begin{abstract}
The present paper is devoted to the statistical study of plasma structures observed at the interface between magnetospheric lobes and the plasma sheet at distances of 15$25 R_{E}$. The majority of the registered structures are bursty earthward ion streams with energies about 10-30 keV known as "beamlets". The paper describes the results of beamlet statistical analysis for different interplanetary magnetic fields (IMF), clock-angles and various magnetospheric conditions. It is shown that the energy of beamlets increases monotonously with a distance from the neutral sheet (NS) during slightly southward IMF and quiet magnetospheric conditions. Under these conditions, beamlets are observed up to 5-6 $R_{E}$ over the NS. In contrast, the beamlet energy decreases with increasing distance from the NS if the IMF is northward, and the region of beamlet observation becomes much wider up to $12 R_{E}$ above the NS. The same (inverse) beamlet energy dependence on the distance from the NS is also registered during active magnetospheric periods.

Yet, the plasma structures of various duration with quasiisotropic ion velocity distributions resembling the plasma of the plasma sheet (PS) were observed in the same region. Statistical study performed for various IMF clock-angles demonstrates the clear difference in the spatial distributions of such plasma structures for two extreme IMF directions. When IMF is primarily northward, PS-like plasma fills the lobe region almost entirely and could be associated with the PS expansions. For primarily southward IMF time intervals, the probability of observing PS-like structures of long duration sharply decreases with the increasing distance from the NS, but PS-like structures of short durations $(<500 \mathrm{~s})$ are distributed almost uniformly up to $12 R_{E}$ above the NS. This feature cannot be described in terms of the PS flapping motions and requires another explanation. Relevance and limitations of the existing models attempting to explain the generation mechanisms of these transient structures are briefly discussed in the concluding part of the paper.
\end{abstract}

Correspondence to: E. E. Grigorenko

(grig@afed.iki.rssi.ru)
Key words. Magnetospheric physics (magnetotail; magnetotail boundary layers; plasma sheet)

\section{Introduction}

Numerous measurements carried out in 1980's demonstrated the existence of the interface between the hot quasi-isotropic Central Plasma Sheet (CPS) and the magnetotail lobe region (Eastman et al., 1984, 1985; Parks et al., 1984; Takahashi and Hones, 1988). This temporal variable transition region was called the Plasma Sheet Boundary Layer (PSBL). The key role of the PSBL in the magnetotail dynamic, an important region of mass, energy and momentum transport, was realized since these early observations. First, it was assumed that the existence of the PSBL can be related to the substorm processes (Winningham et al., 1975) and only later it was proved that the PSBL is a permanent feature of the magnetotail presented even during the extended quiet periods (Eastman et al., 1984).

The PSBL has no well-defined boundaries (Grigorenko et al., 2000) and consists of complex plasma structures including fast earthward (> $400 \mathrm{~km} / \mathrm{s}$ ) flows (Lui et al., 1983; Eastman et al., 1984; Baumjohann et al., 1988). Bidirectional beams are observed with higher densities further inside the PSBL (Parks et al., 1998) and then they evolve into the hot isotropic CPS component.

High velocity ion beams are observed in a rather wide region including magnetotail lobes (Baumjohann et al., 1988; Orsini et al., 1987). Their distribution function was first reported in the experiment by De Coster and Frank (1979) and theoretically explained by Onsager et al. (1990); Büchner and Zelenyi (1990); Zelenyi et al. (1996). According to the theory, such high velocity localized ion beams (beamlets) are the result of nonadiabatic plasma acceleration in the spatially limited regions of the distant magnetotail. A first attempt to make a kinetic modelling of the complicated mantle plasma acceleration in the magnetotail (Ashour-Abdalla et al., 1993) also revealed that the PSBL could be filled by spatially lo- 


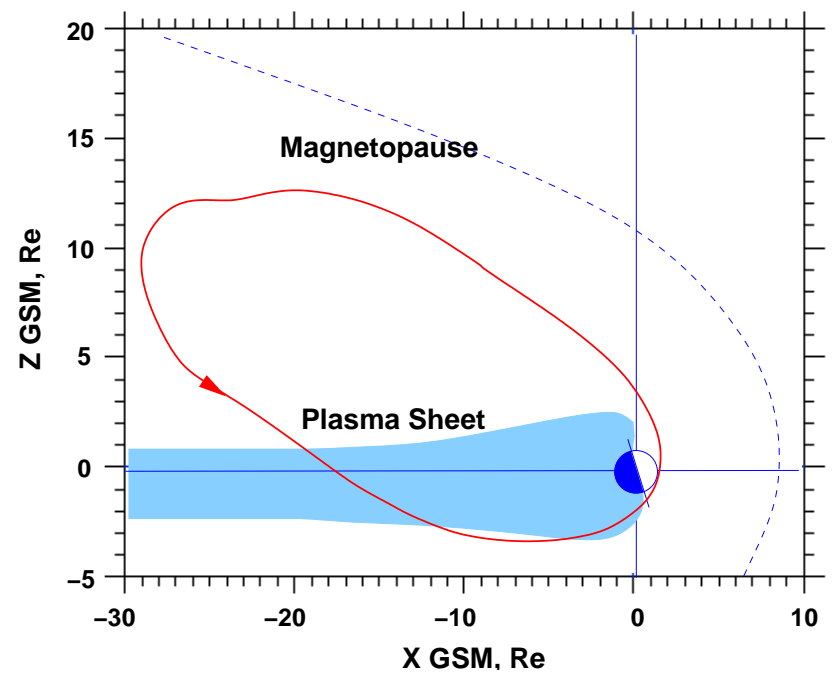

Fig. 1. An example of the Interball-1 (Tail Probe) orbit passing near the midnight meridional plane in winter season.

calized earthward and tailward streaming ion beams. Other theoretical models (Chen et al., 1990; Onsager et al., 1991; Moses et al., 1993) also gave their explanations for the complicated structure of accelerated ion beams in the phase space (see Sect. 6).

Independently, the observations of plasma structures, resembling the "scraps" of the plasma sheet (PS) material (i.e. plasma structures detached from the main body of the PS) and having no pronounced bulk velocities were reported for the PSBL and the adjacent lobe regions (Himenez et al., 1984; Belova et al., 1987). Observation of such "scraps" could be related to the PS flapping motions. But such plasma structures can also be formed as a result of magnetospheric convection changes (Ogino et al., 1994) or due to the development of a large-scale instability at the edge of the PS (Belova et al., 1987; Milovanov et al., 2000).

The Interball-1 spacecraft performed observations in the lobes - PS interface during 1995-2000 time interval. The data discussed in the present paper were obtained in the 1995-1998 years. The aim of this study is to answer the following questions:

1. What types of plasma structures are observed in the PSBL and magnetotail lobes?

2. What is the spatial distribution of the various types of plasma structures under different interplanetary conditions and under different levels of magnetospheric activity?

3. How do the main characteristics of the plasma structures (i.e. energy, duration and so on) depend on the location of the observational point and on the external conditions?

Then we will make an attempt to find the specific characteristics of the plasma properties of these structures which could clarify the physical mechanisms of their formation.

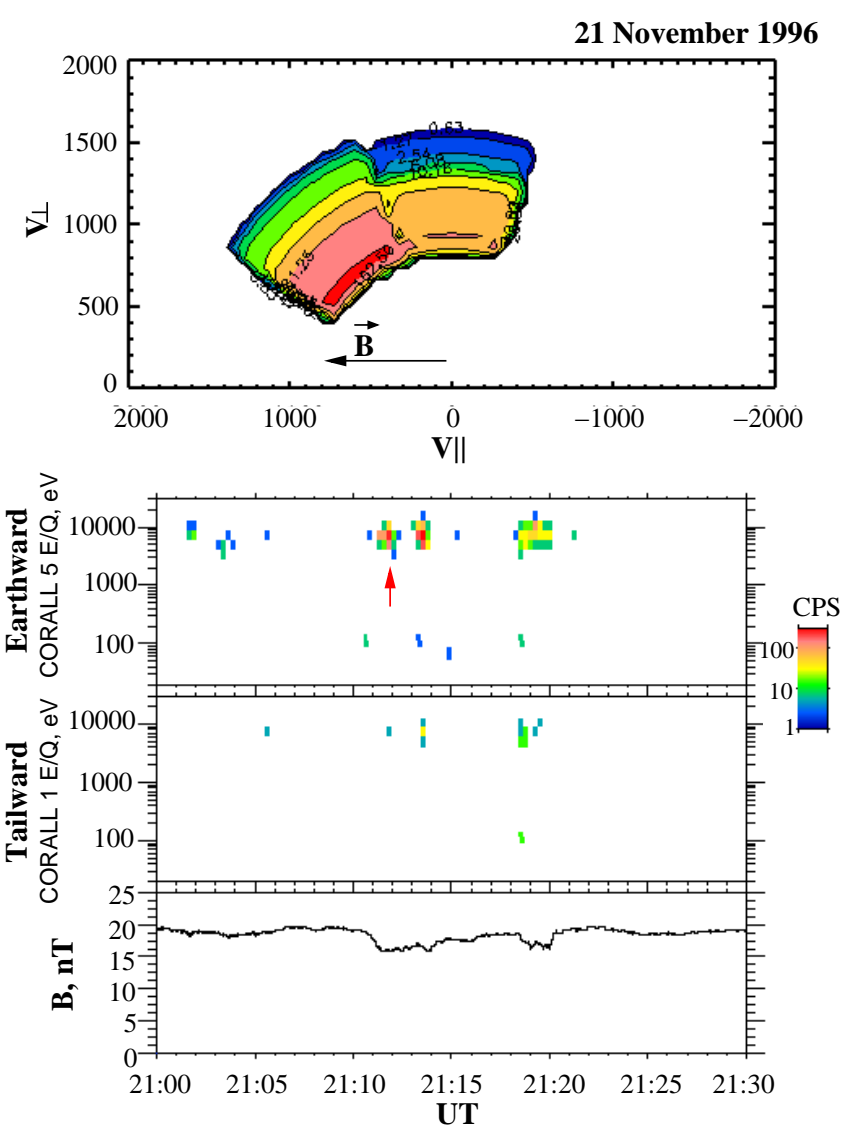

Fig. 2. An example of an isolated beamlet. The lower 3 panels show from top to bottom: E-T spectrogram of earthward ions, E-T spectrogram of tailward ions, the magnitude of the magnetic field. The red arrow marks the beamlet event. The $V_{\perp}-V_{\|}$distribution function of the marked beamlet obtained for $3.8 \mathrm{~s}$ is shown in the top panel.

The organization of the paper is as follows: in Sect. 2, the Interball-1 orbit and the data used in our analysis are described. In Sect. 3, an overall description and examples of observed plasma structures are presented. In Sects. 4 and 5, we demonstrate the statistical characteristics of beamlets and PS-like plasma structures, respectively. Section 6 is devoted to the discussion of possible physical mechanisms of such "plasma regime" formations. The conclusion is presented in Sect. 7.

\section{Instrumentation}

The Interball-1 spacecraft was launched in 3 August 1995 to study mass, energy and momentum transport in the critical regions of the solar wind/magnetospheric system. Initial apogee and perigee of the orbit were $30 R_{E}$ and $800 \mathrm{~km}$, respectively, and the orbit inclination was $63^{\circ}$. The satellite spins around the axis pointing to the Sun with a period of $118 \mathrm{~s}$. An example of one real Interball-1 orbit is shown in Fig. 1. At each of the tail orbits the satellite crossed the magnetotail lobe-PSBL-PS interface region. 


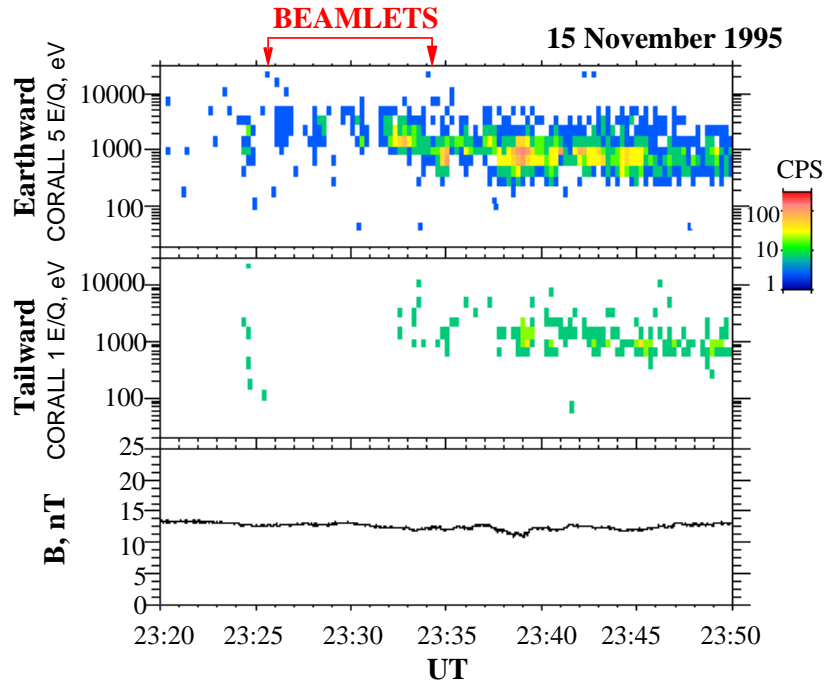

Fig. 3. An example of "attached" beamlet. The figure layout is the same as Fig. 2 except for distribution.

The present study was undertaken for the midnight region of the tail. We analyzed 42 PSBL crossings that occurred inside $\left|Y_{\mathrm{GSM}}\right|<7 R_{E}$ interval. We used vector magnetic field data and plasma measurements from the MIF magnetometer (Klimov et al., 1997) and CORALL (Yermolaev et al., 1997) ion spectrometer, respectively. The ion spectrometer CORALL allowed one to obtain 3-D distribution (without mass selection) for about $2 \mathrm{~min}$ (spacecraft spin period). The individual energy spectrum in the energy range $0.03-25 \mathrm{keV}$ was collected for $3.6 \mathrm{~s}$. The azimuthal resolution of the instrument was about $10^{\circ}$, but polar resolution (elevation from the solar-spacecraft line) was about $40^{\circ}$. The IMF data monitored by the Wind spacecraft were obtained via CDA WEB (http://cdaweb.gsfc.nasa.gov). Wind data were shifted for solar wind propagation time between Wind and Interball-1. Geomagnetic activity indices Dst, $K_{p}, A E$ were obtained from the Geomagnetic Data Service (http://swdcdb.kugi.kyoto-u.ac.jp/wdc/Sec3.html).

\section{Observations of Plasma Structures.}

High velocity ion beams (beamlets) moving earthward (more rarely tailward) are indentified in spectrograms as bursty plasma events along with the $|B|$ depression. The typical event duration is not greater than $2 \mathrm{~min}$. All events were registered when $\left(B_{X} /|B| \sim 1\right)$, i.e. in the northern lobe. Figure 2 shows the example of the isolated beamlet observed far from the PS region. Often, beamlets are observed in the regions adjacent to the PS, as it is shown in Fig. 3. This fact conforms the concept that the external part of the PS consists of the short intermittent ion beams (Ashour-Abdalla et al., 1993). All beamlets have a characteristic velocity distribution function of a "lima-bean" shape (De Coster and Frank, 1979; Onsager et al., 1991; Zelenyi et al., 1996; AshourAbdalla et al., 1996) shown in two panels of Fig. 2. More-
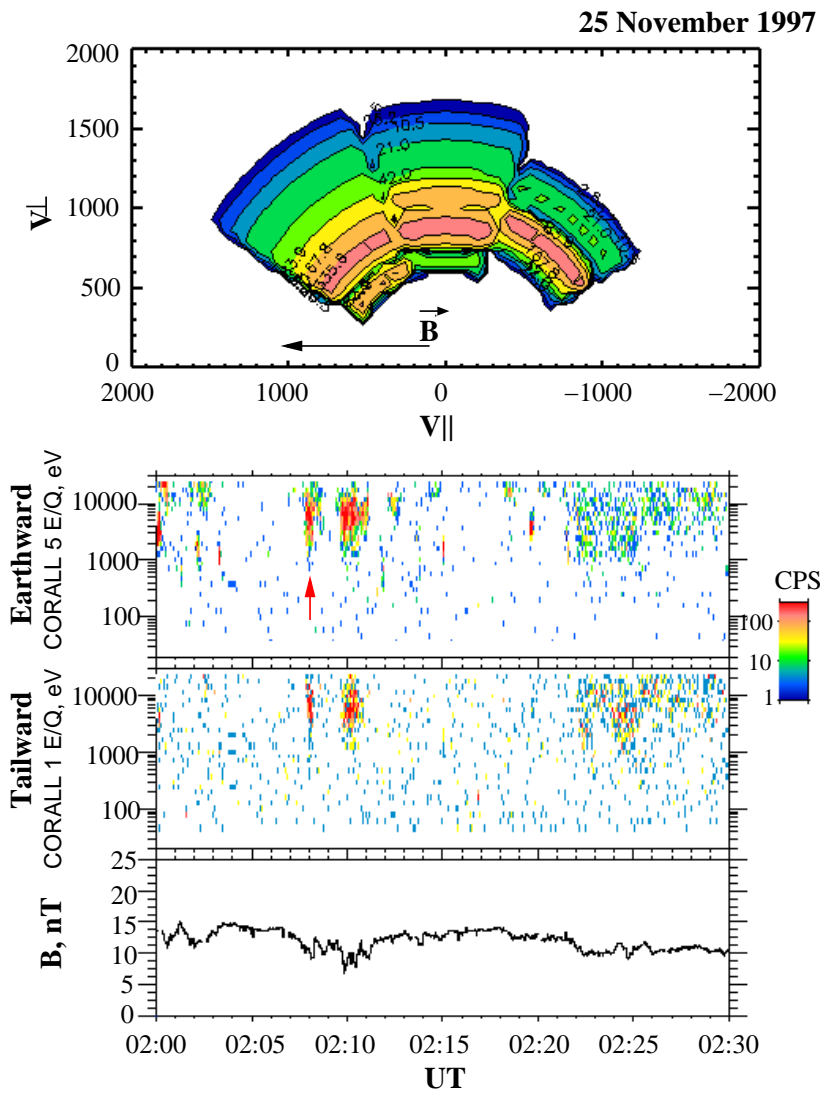

Fig. 4. The same as Fig. 2 showing a bidirectional ion beam observation.

over, in some cases, a combination of earthward and tailward streaming (reflected) beamlets is observed. In these cases, the distribution function has a ring-like shape, as in Fig. 4. We have analysed 83 cases of beamlet observations for different interplanetary and magnetospheric conditions. The results of the study are discussed in Sect. 4. At the same time and almost at the same distances from the tail mid-plane, we observe quasi-isotropic plasma structures of various durations, which are accompanied by the magnetic field depression due to a diamagnetic effect. The typical experimental example of such a plasma structure is illustrated in Fig. 5. In contrast to beamlets, the ion velocity distribution function of such plasma events reveals nearly a quasi-isotropic but complex structure. The complexity of the distribution function could be due to transient effects or due to low statistics. The main properties of such plasma structures, e.g. temperature $\left(T_{i}>1000 \mathrm{eV}\right)$, and an almost isotropic velocity distribution makes them resemble the plasma of the PS. So we will call them "PS-like plasma structures". Beamlets and PS-like plasma structures are clearly distinguished by their bulk velocity vectors. While the beamlet bulk velocity is primarily parallel to the magnetic field, plasma clouds may have rather high velocities across the magnetic field (in $Y$ and/or $Z$ direction). The statistical analysis of such structures is presented in Sect. 5.

Now, we cannot say definitely if these structures are de- 


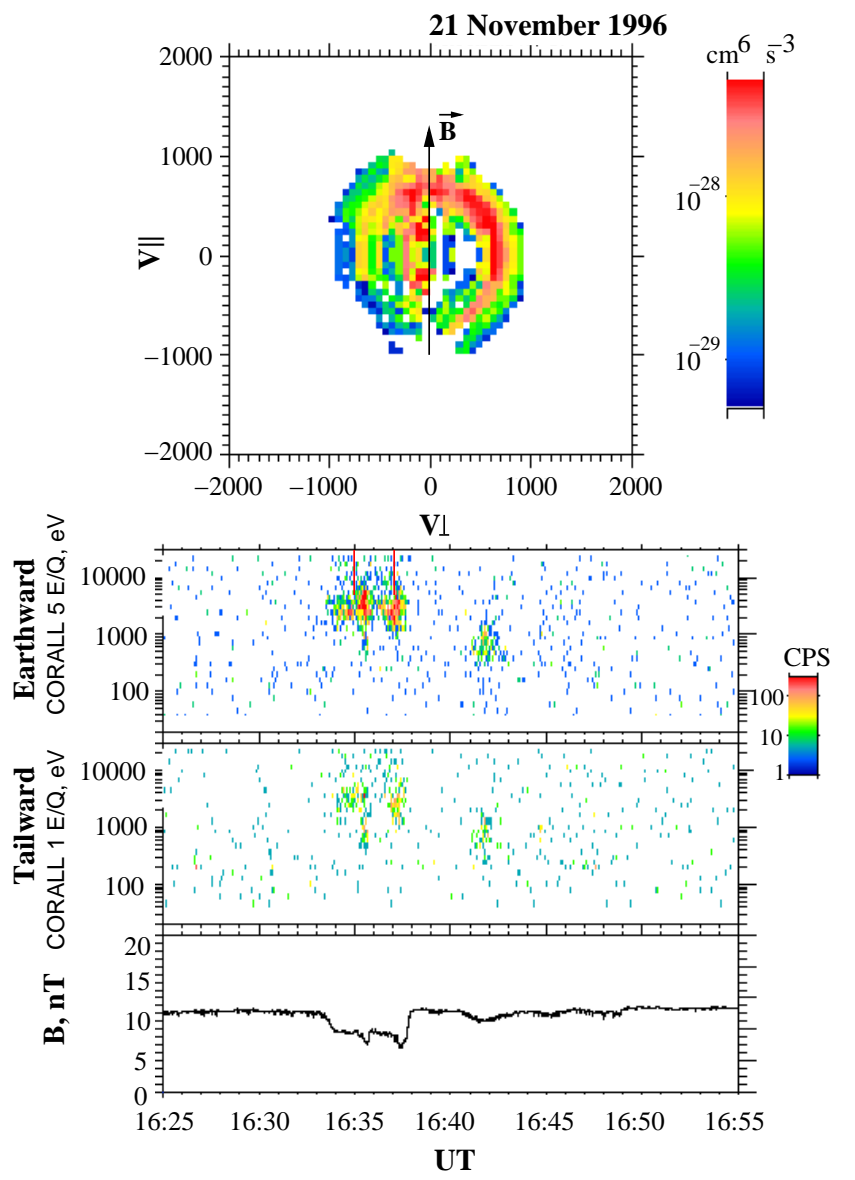

Fig. 5. An example of PS-like structure (plasma filament) observation. Figure layout is the same as in Fig. 2.

tached from the main body of PS or their observations are related to the episodic excursions to the PS due to its flapping motions. In Sect. 6, we will make some speculations about the origin of such PS-like plasma structures.

\section{Statistical analysis of beamlet observations}

In this section we perform the statistical analysis of beamlet observations for different magnetospheric and interplanetary conditions. To characterise the magnetospheric conditions, we use the $K_{p}$ and $A E$ geomagnetic indices. The $K_{p}$ index is a planetary $K$ index, which, in turn, is obtained as the mean value of the disturbance levels in the two horizontal field components at a given station that measures the magnitude of disturbances caused by the phenomena other than the diurnal variation and the long-term component of the stormtime variations, averaged for $3 \mathrm{~h}$. The $K_{p}$ index is obtained from the $K$ index by specific calculations and an averaging over 13 standard subauroral stations distributed over the entire Earth. Since ionospheric currents in the auroral zone considerably influence the magnetic field near the Earth, we also use the $A E$ index for our analysis. The $A E$ is the auroralelectrojet index which was defined to obtain a measure of the

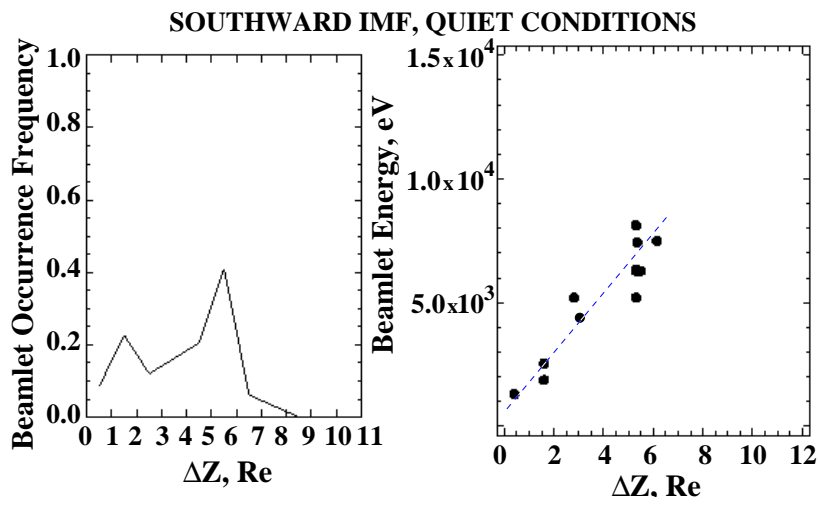

Fig. 6. Left panel: beamlet occurrence frequency versus the nominal distance from the neutral sheet $(\Delta Z)$ for quiet magnetospheric conditions under the southward IMF. Right panel: scatterplot of beamlets energy (i.e. energy associated with the field-aligned velocity of the beam) versus $\Delta Z$.

strength of the auroral electrojets relatively uncontaminated by the effects of ring current.

We have divided all PSBL crossings into 3 groups according to the corresponding magnetospheric and interplanetary conditions. Namely:

1. southward interplanetary magnetic field (IMF), quiet conditions $\left(K_{p}<1+, A E \leq 50 \mathrm{nT}\right)$;

2. southward IMF, disturbed conditions $\left(K_{p} \geq 1+, A E>\right.$ $50 \mathrm{nT}$ );

3. northward IMF, quiet conditions (the same as in group 1).

Assuming the mechanism of the beamlet formation is the non-adiabatic acceleration in the distant magnetotail (Ashour-Abdalla et al., 1993, 1995; Moses et al., 1993; Harold et al., 1998), first of all, we have selected the cases of beamlet observations when the IMF was southward (the cross-tail electric field was definitely directed from down flank to the dusk, which provides the favourable acceleration conditions in the neutral sheet) and the magnetosphere was quiet enough $\left(K_{p}<1+, A E \leq 50 \mathrm{nT}\right)$ to clarify the physics of energization without the disturbing factors. We have found 15 cases which satisfied these criteria. All of these beamlets were observed outside the current sheet $\left(B_{X} /|B| \sim 1.0\right)$. Figure 6 (left panel) shows the statistics of the beamlet occurrence frequency versus the distance $\Delta Z$ to the position of the NS. To avoid a satellite velocity effect, the "beamlet occurrence frequency" was calculated as the number of beamlet events encountered inside the $1 R_{E}$ bin along $\Delta Z$ axis normalized to the time required to cross this bin. The probability of observing beamlets increases with the distance from the NS and drops down when $\Delta Z$ reaches $6 R_{E}$. In the right panel of Fig. 6, beamlet energies (i.e. energy associated with the field-aligned velocity of the beam) versus $\Delta Z$ are presented. The energy of the beamlet increases with the distance from the NS. Such an energy profile is predicted by 

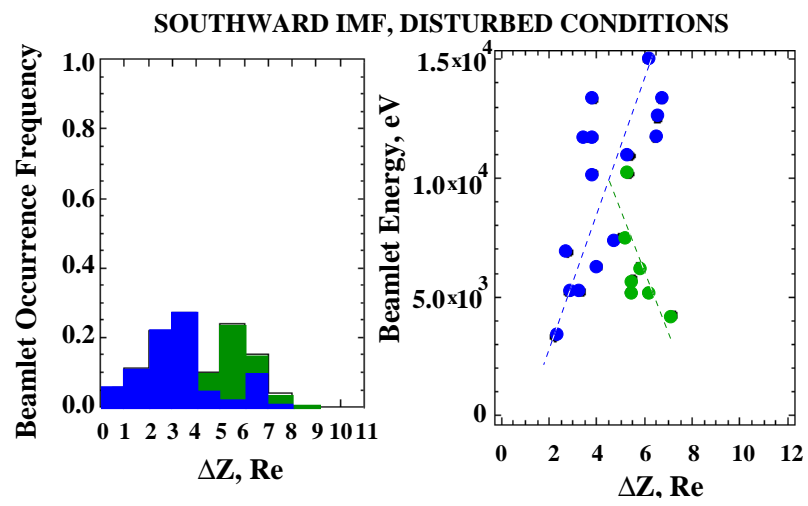

Fig. 7. Left panel: beamlet occurrence frequency versus $\Delta Z$ for disturbed magnetospheric conditions under southward IMF. Two distributions displayed by blue and green correspond to two branches of energy profile shown in the right panel. Right panel: Scatterplot of beamlet energy versus $\Delta Z$. Two different beamlet populations are marked by blue and green.

theory (Onsager et al., 1991; Ashour-Abdalla et al., 1993) and we call it "normal beamlet velocity dispersion".

During the active magnetospheric periods $\left(K_{p} \geq 1+\right.$, $A E>50 \mathrm{nT}$, IMF is southward, 28 registered events), the spatial distribution of beamlet occurrence frequency distribution is more spread out and has two maxima (see Fig. 7, left panel). Beamlet energy spatial distribution is now more complicated (Fig. 7, right panel). One can see here two beamlet populations marked by different colours with normal and inverse velocity dispersions. Note that the maximum beamlet energy under the disturbed magnetospheric conditions is two times higher than the maximum energy during quiet periods. The main part of these beamlets was observed when $K_{p}$ was moderate $(<3)$; neither altitude of beamlet observation nor beamlet energy are dependent on the value of $K_{p}$.

The last group of selected events ( 24 cases) was obtained when IMF was northward and the magnetosphere was quiet. Fourteen cases corresponding to northward IMF and disturbed magnetospheric conditions were excluded as intermediate cases. Figure 8 (left panel) shows that beamlets were observed at higher distances above the NS. The right panel of this figure clearly displays the inverse beamlet energy dispersion profile. In Sect. 6, we will discuss all beamlet distributions presented here.

\section{Statistical distributions of PS-like plasma structures}

The spatial distribution of PS-like structures with a duration of more than 2 min is shown in Fig. 9. The observation probability of such structures versus the distance from NS $\Delta Z$ for two external IMF clock-angles is presented in panels (a) and (b), respectively. The bottom panel shows the scatter plot of $B_{X} /|\boldsymbol{B}|$ versus $\Delta Z$, to indicate the transition from the CPS to the lobe region. The observation probability was calculated as a ratio of total duration of PS-like plasma observations when the satellite was in a particular $\Delta Z$ bin of

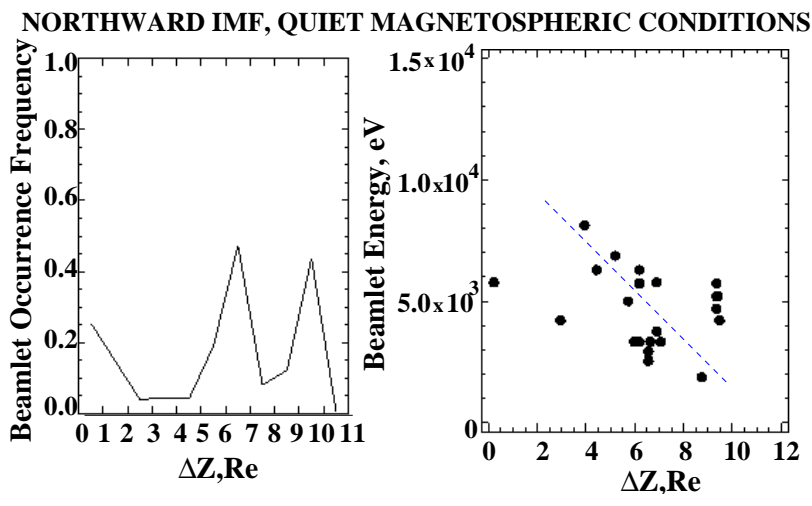

Fig. 8. The same as Fig. 6 obtained during quiet northward IMF time intervals.

$1 R_{E}$ width to the total time which the satellite spent inside this $\Delta Z$ bin. Contrary to beamlets, the probability profile of PS-like plasma structures has no clear maximum but decreases gradually with $\Delta Z$. When the IMF is northward, the spatial distribution of PS-like plasma structures observed in the lobe region looks like the PS extension filling the lobes with decreasing probabilities. But when the IMF is southward, the distribution has a long, weak but almost constant tail observed in the 7-17 $R_{E} \Delta Z$ interval.

The general pattern of the distribution of probability to observe PS-like structures in the $\Delta Z$ - clock-angle plane is shown in Fig. 10a. Since the electric potential drop across the polar cap is a fundamental measure of the coupling between the solar wind and the Earth's magnetosphere, it would be useful to display the PS-like structures distribution versus some empirical value, which correlates with the polar cap potential drop. Such an empirical value is the "reconnection electric field", $E_{R}=\left|\boldsymbol{V}_{s w} \times \boldsymbol{B}_{\mathrm{IMF}}\right| \cdot \sin ^{4}\left(\frac{\Theta}{2}\right)$ (Eriksson et al., 2000; Wygant et al., 1983). Here, $\Theta$ is the IMF clock-angle, and $\boldsymbol{V}_{s w}$ is the solar wind velocity. The $2 \mathrm{D}$ distribution of the probability of observing plasma filaments versus $\Delta Z$ and $E_{R}$ is displayed in Fig. $10 \mathrm{~b}$.

The region occupied by PS-like plasma structures expands while the IMF clock-angle is decreasing. When the IMF is exactly northward (clock-angle $=0$ ), PS-like structures fill almost the entire lobe region up to $\Delta Z=12$. This feature can be associated with $\Theta$-aurora (Frank et al., 1986). Figure 10b shows the same properties of the PS and PS-like structures even more clearly. Here, the dramatic expansion of the PS is seen at $E_{R}<100 \mu \mathrm{V} / \mathrm{m}$. If $E_{R}>600 \mu \mathrm{V} / \mathrm{m}$, then the probability of observing PS is very low even at $\Delta Z=0$. It looks like thinning of the PS or a fragmentation of the PS due to substorm activity. Note that the weak constant tail of PS-like structures seen in Fig. 9b is under the low colour limit in Fig. 10. We will discuss the nature of PS-like structures in the next section. 

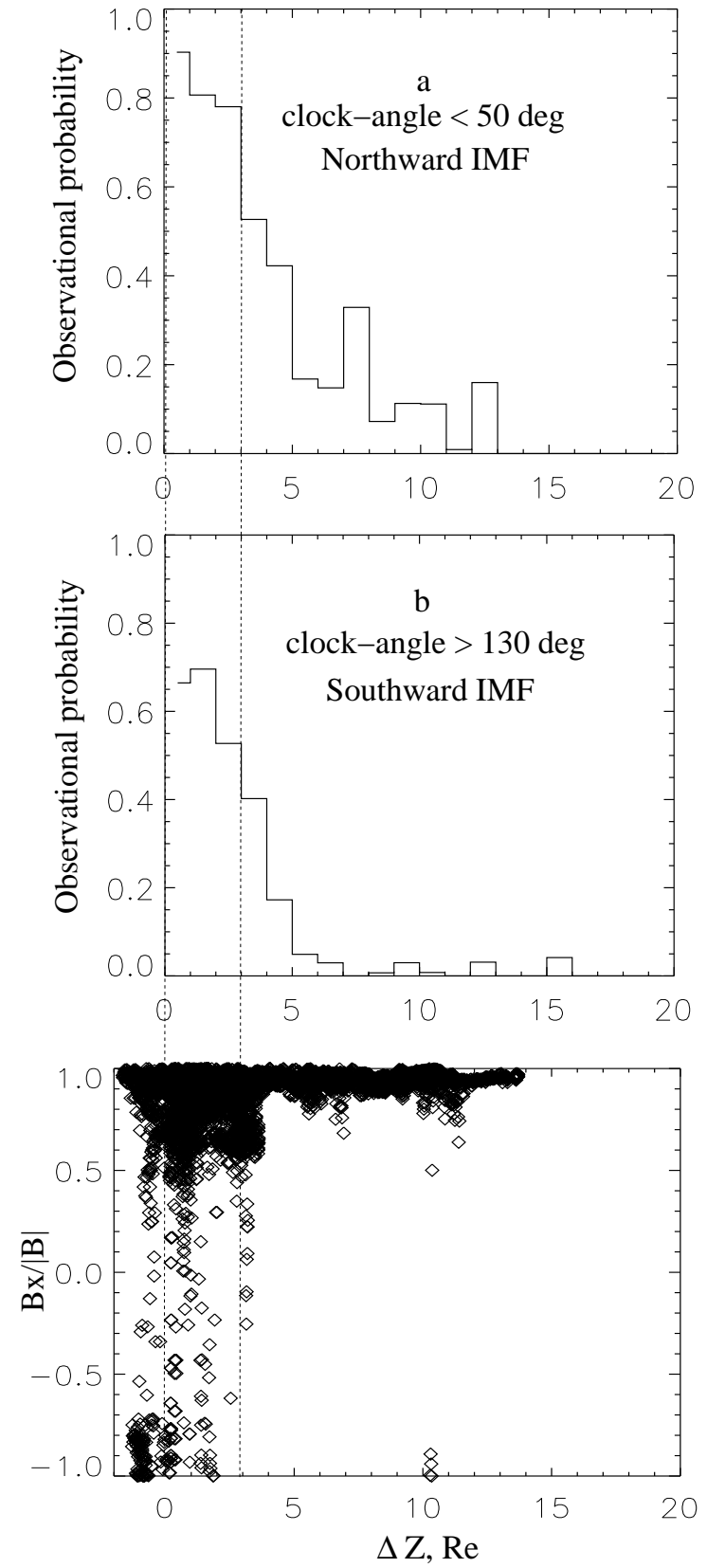

Fig. 9. Probability to observe PS-like structures versus $\Delta Z$ for northward IMF (a) and for southward IMF (b). The lower panel shows the scatterplot of $B_{X} /|\boldsymbol{B}|$ to demarcate the PSt boundary which is shown by dashed vertical line.

\section{Discussion}

Since an appearance of beamlets and other transient structures reflects very deep properties of the nonlinear selfconsistent plasma configuration of the Earth's magnetotail, numerous theoretical efforts have been made to explain the plasma structuring in velocity and/or configurational space. The idea of explaining such plasma structures in the course of non-adiabatic particle acceleration in the distant magne- totail (Lyons and Speiser, 1982) originates from the revisiting of Speiser model of particle motion at the end of 1980's (Chen and Palmadesso, 1986; Büchner and Zelenyi, 1989). Later, the resonance nature of the non-adiabatic interaction has been revealed (Burkhart and Chen, 1991; Büchner, 1991; Ashour-Abdalla et al., 1993). Independence of the so-called parameter of adiabaticity

$\kappa=\frac{B_{n}}{B_{0}} \sqrt{\frac{L}{\rho_{0}}}$,

(where $\rho$ is a particle's Larmor radius, $L$ is the current sheet thickness, $B_{0}$ is the value of the magnetic field in the lobe, $B_{n}$ is the normal component of the magnetic field at the site of particle interaction with the current sheet), particle motion in a prescribed field (used in all these models) could experience either strong scattering or follow the almost unperturbed classical Speiser orbit. This dependence is very nonmonotonous and could, therefore, result in a phase space structuring. Independently from different grounds, Moses et al. (1993) also considered peculiarities of non-adiabatic particle motion in a vicinity of $X$-line (also in a prescribed field) and found a partitioning (even fractalization) of the phase space of accelerated population. Although Moses et al. (1993) did not explicitly invoke $\kappa$ - resonance arguments, the physics under consideration was essentially the same. A 2-D test particle model by Ashour-Abdalla et al. (1993), assuming the weak gradient in $B_{Z}(X)$ (i.e. $\kappa(X)$ dependence) and a uniform cross-tail electric field, predicts the formation of resonance regions at a number of locations in the distant magnetotail and produces a spatially fragmented pattern of the PS plasma.

On the other hand, Harold et al. (1998) recently found that beamlets disappear if instead of the uniform dawn-dusk electric field, the electric field taken from the MHD model is used for the trajectory calculations. However, the formation of spatial structure of any given beamlet is a local process and if the profile of $E_{y}$ is nonuniform (and even strongly nonuniform), it might influence the energy of beamlets and their spatial distribution, but not the fact of their existence. Another point of Harold et al. (1998) is that beamlets also disappear if in their (nonself-consistent) calculations, the thickness of the current sheet was taken close to $\rho_{i 0}-$ ion Larmor radius in a lobe field $B_{0}$. From our point of view, the ions which form beamlets carry the main portion of the cross-tail current at the location of their acceleration and, therefore, their dynamics in the course of acceleration (strong or weak scattering) determines the overall self-consistent structure of the magnetotail configuration, as was recently shown by Sitnov et al. (2000) and Zelenyi et al. (2000). So the processes of beamlet generation could adequately be described only by the self-consistent approach. The first analytical attempt to formulate the self-consistent theory of forced current sheets, taking into account the jumps of quasi-adiabatic invariant, was made only recently by Zelenyi et al. (2001).

Another approach, advocated in papers by Burkhart and Chen (1991), Chen et al. (1990), Holland et al. (1999) as- 


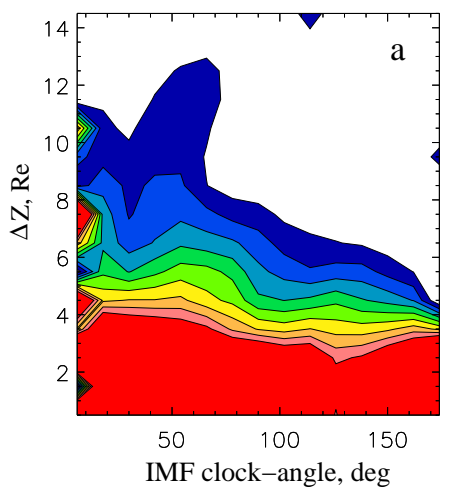

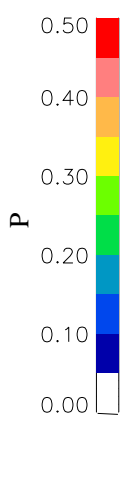

Fig. 10. (a) Color-coded distribution of probability $P$ to observe PS-like structures versus $\Delta Z$ and IMF clock-angle. (b) The same versus $\Delta Z$ and reconnection electric field $E_{R}=\left|\boldsymbol{V}_{s w} \times \boldsymbol{B}_{\mathrm{IMF}}\right|$ $\sin ^{4}\left(\frac{\Theta}{2}\right)$; here, $\boldsymbol{V}_{s w}$ is the solar wind velocity, $\boldsymbol{B}_{I M F}$ is the IMF, and $\Theta$ is the IMF clock-angle. The $E_{R}$ is marked in microvolts per meter. sumed that the resonances are produced locally at a given $X$ and due to $\kappa(v)$ dependence. The authors also declared the finding of some experimental manifestations of such resonances in the velocity space, i.e. isolated multiple velocity peaks (Chen et al., 1990; Holland et al., 1999). But this approach completely neglects the velocity filter effect whose importance has been thoroughly investigated in a series of papers by Green and Horwitz (1986); Onsager et al. (1990, 1991). The point is that the phase space structures with different velocities formed locally will be subjected to velocity dispersion in the course of their propagation from the formation site to the observation site. As a result, in a given region where our observations are made, we will never see the velocity peaks formed in the one certain location downstream in the magnetotail, but rather a combination of velocity structures formed at different locations near the tail mid-plane (see below).

Although in papers by Onsager et al. $(1990,1991)$ the mechanism of beam formation has not been discussed, they clearly demonstrated the importance of the velocity filter mechanism in forming the PSBL plasma distributions even in a simple 1-D model with $B_{n}(X)=$ const. Results of AshourAbdalla et al. (1993) incorporated both the velocity filter effect and another so-called "place of birth" effect, taking into account that an additional cause of dispersion is related to the dependence of the energy $\Delta W$ acquired in the course of non-adiabatic energization on the position of the acceleration site

$\Delta W(X)=2 M\left(\frac{c E}{B_{Z}(X)}\right)^{2}$.

As it has already been reported, beamlets have a typical "lima-bean" shape of the velocity distribution function. The sketch of the formation of such a distribution is presented in Fig. 11. Due to the velocity filter effect produced by the global magnetotail convection in the $Z$ direction, different parts of the distribution function measured at the given point should be generated at the different sites of the magnetotail (points A and B in Fig. 11).

If the convection is directed downward (as in Fig. 11), then ions with smaller field-aligned velocities should be generated in the site far enough (in point B, for instance) to be registered at the same point as the ions with higher parallel velocities (emitted from the point A). So the resulting beamlet distribution function measured at the given point of observation is the sum of the pieces cut out from the beamlet distributions generated at the different distances from the Earth. Figure 11 presents the particular case when the $B_{Z}$ component of the magnetic field decreases monotonously with the distance from the Earth. Hence, particles with a large pitch-angle and low $V_{\|}$(generated farther in the tail from the point of observation) will have larger energy, creating "lima-bean" distribution at the observation site. Note that any $B_{Z}$ profile in the region of beamlet generation causes arcshaped ion distribution with various $V_{\|} / V_{\perp}$ ratio. Although our sketch in Fig. 11 resembles the one in Fig. 3 of Onsager et al. (1991), the physics involved in the explanation of the lima-bean shape is somewhat different. While Onsager et al. (1991) considered the formation of such distributions from the "bursty-like" local source ("all particles that travel from point A must do so in the same time", according to their argumentation), we consider that the distributed quasi-permanent source and particles with different energies registered at the observer location are coming from the different sites in the magnetotail.

Our assumption about the importance of both velocityfilter and "place of birth" effects is supported by our analysis of statistical spatial beamlet distributions. The results shown in Fig. 6 already suggest that (at least for southward IMF and relatively quiet conditions) due to the clear dependence of beamlet energy on $\Delta Z$, there should exist a well pronounced statistical decrease in $B_{Z}(x)$ with distance from the Earth (see Eq. 2).

For further discussion we present our results as a 2Dhistogram showing spatial beamlet energy distribution as a function of IMF clock-angle (Fig. 12). The clock-angle was $1.5 \mathrm{~h}$ averaged before the encounter with the beamlet. We divided the total interval of IMF clock-angles into 3 groups marked by the letters "C", "D", and "N" in Fig. 12. The cases lying inside each of these sub-intervals ("C", "D", or "N") are exactly the same ones as included in our statistics shown in Figs. 6-8. 


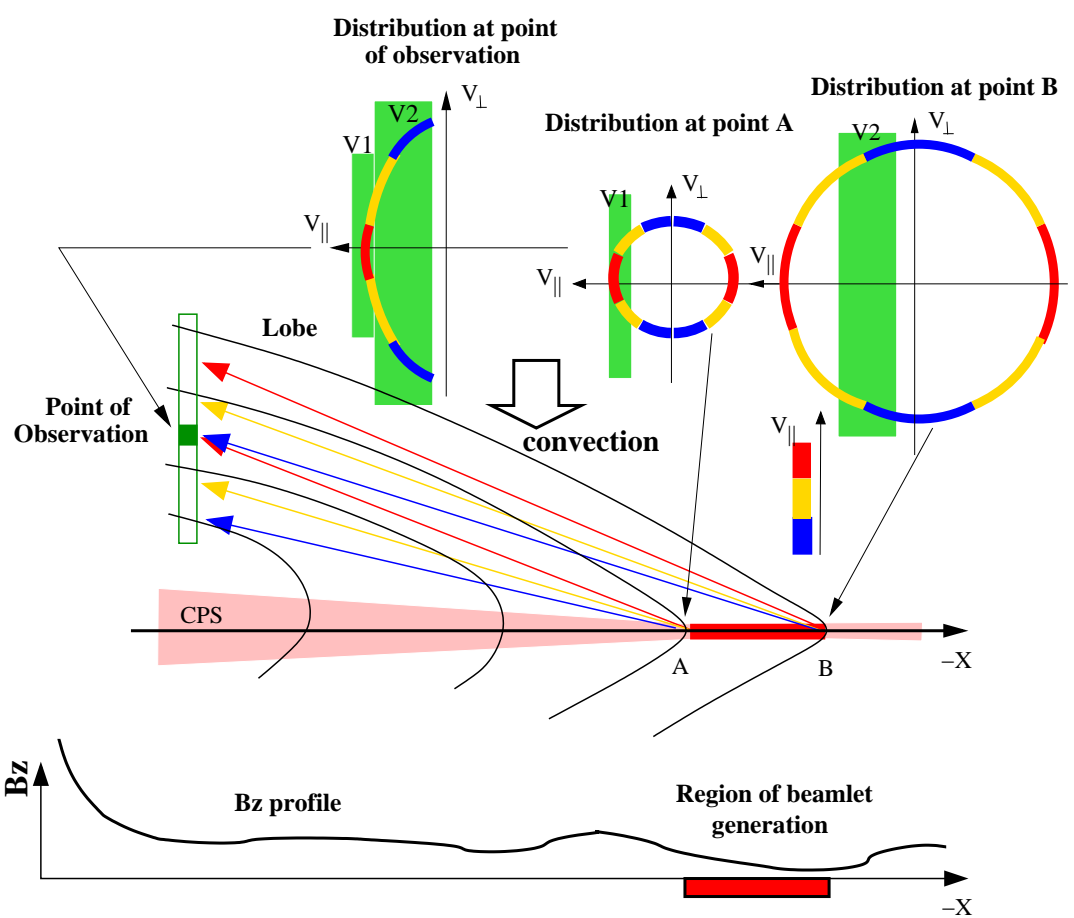

Fig. 11. The sketch showing the formation of the beamlet. The horizontal red bar shows the possible beamlet generation region. Each point of this region generates a ring-like distribution. Due to the convection filter, only specific $V_{\|}$can reach the point of observation (green rectangular). Different color arcs in the distributions show different $V_{\|}$. Color arrows show the appropriate trajectories of varying $V_{\|}$. The observed distribution function is the collection of particles accelerated at the point $\mathbf{A}$ with the $V_{\|}$in the "V1" band, and particles accelerated in the point $\mathbf{B}$ with $V_{\|}$in the " $V 2$ " band.
1. The "normal" beamlet energy dispersion is observed within clock-angle interval of $90^{\circ}-110^{\circ}$ that corresponds to the moderate values of the dawn-dusk crosstail electric field (i.e. low substorm probability). In this case, $B_{Z}$ should statistically decrease tailward practically monotonously, and beamlets with higher energies are generated farther from the Earth and observed at higher $\Delta Z$ (see Fig. 13, top panel) that corresponds to their acceleration in the vicinity of the distant $X$-line;

2. When the clock-angle is in the range of $110^{\circ}-130^{\circ}$, beamlet energy increases, but at the same time, one can begin to observe beamlets at the lower $\Delta Z$. This can be associated with the $B_{Z}(x)$ decrease over a wide magnetotail region (increase in the cross tail current). Thus, the beamlet generation site becomes wider and approaches the Earth;

3. When the IMF is primarily southward (that corresponds to clock-angles in the $130^{\circ}-180^{\circ}$ interval), beamlets with the highest energies are registered at the lowest $\Delta Z$. The inverse energy dispersion is seen in the 4$8 R_{E} \Delta Z$ range. (Compare with the green points in Fig. 7). Such distribution may be the result of substorm activity and may be explained by the new $X$-line formation closer to the Earth. In this case, the negative $d B_{Z} / d Z$ profile can be formed behind the new $X$-line (Zelenyi et al., 1990), and the inverse beamlet dispersion can be generated according to the scenario shown in the lower panel of Fig. 13;

4. The inverse energy dispersion is observed also when the IMF is generally northward (clock-angle is $0^{\circ}-90^{\circ}$ (see
Figs. 8 and 12). In this case, beamlets are registered up to very high distances from the NS (up to $\Delta Z \sim 11 R_{E}$ ) and have relatively small energies $(\leq 8 \mathrm{keV})$.

The reverse convection (Ogino et al., 1994) directed from the NS toward the magnetopause is expected when IMF is exactly northward. This process leads to the inverse beamlet velocity dispersion. Also, although less probable, is that negative dispersion can be related to the formation of a negative $d B_{Z} / d X$ gradient under northward IMF.

We see that both the lima-bean shapes of the individual beamlet distribution functions and statistical spatial beamlet distributions could be explained in terms of the model taking into account both velocity filter effects and peculiarities of non-adiabatic (Speiser) acceleration (Eq. 2). It is more difficult to explain the "bursty" appearance of beamlets. It may indicate that the resonance conditions for beamlet acceleration are fulfilled at the given site only for a short time. Alternative explanations invokes the flapping motions of the spatially structured magnetotail. Time scales estimated by Ashour-Abdalla et al. (1995) from the latter assumption are of the order of 1-2 min which conforms with our observations. In reality, spatial structuring could be accompanied by the transient effects considered in the self-consistent generalization of the above mentioned model to the dynamic case (Peroomian et al., 2000). From our single-point observations, it is very complicated to distinguish in each case the spatial or temporal origin of the bursty beamlet manifestations. Hopefully, 4-point Cluster-II observations will clarify this very important issue.

The mechanism of the formation of the PS-like plasma structures is even more poorly understood. When the IMF 

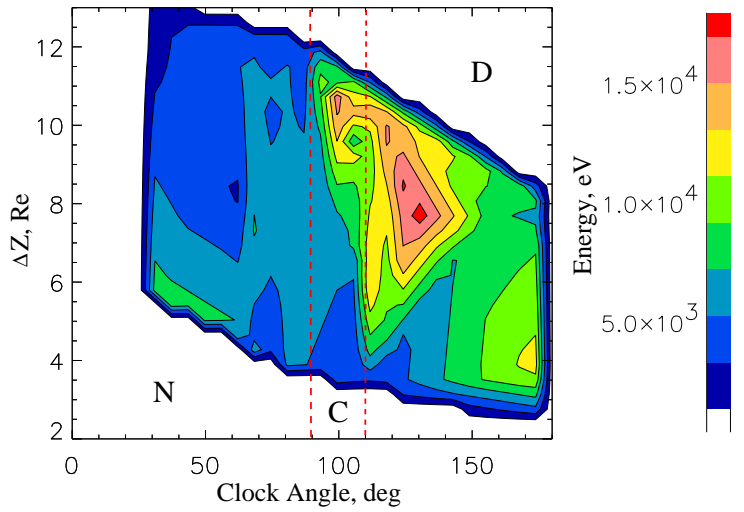

Fig. 12. Color-coded beamlet energy histogram displayed in the IMF clock-angle $-\Delta Z$ plane. Two vertical lines divide the clockangle range into three zones: $\mathrm{D}$ - disturbed conditions in the magnetosphere, $\mathrm{C}$ - quiet conditions, and $\mathrm{N}$ - the northward IMF direction.

turns northward, the change in the direction of plasma convection (Ogino et al., 1994; Walker et al., 1999) can result in the PS expansion to the magnetotail lobes. The weak wings of the distribution seen in Figs. 9 and 10 could be explained by the flapping motions of the PS or by plasma structures (fillaments) detached from the main body of PS. To clarify the problem, it is necessary to study the temporal features of PS-like plasma structures.

In Fig. 14, the probability of observing a PS-like plasma structure is presented as a function of $\Delta Z$ and the event duration. The distributions show that:

1. For the northward IMF, the observed spectrum of PSlike structure durations is distributed almost uniformly over various $\Delta Z$. This may be explained as a result of the PS motion as a whole entity;

2. For the southward IMF, only PS-like structures with the durations $<500 \mathrm{~s}$ are registered with the equal probability over the entire $\Delta Z$ range. The events with the longer durations are observed only within $\Delta Z<4 R_{E}$. Hence, we can conclude that the appearance of the short PSlike plasma structures is caused by the physical mechanism, different from the ones operated for northward IMF. Standard reconnection models, which provide the plasma jetting along the separatrix between open and closed field lines (i.e. along PSBL edge), could not explain such observations.

One possible explanation may be the decays of the PS extensions to the lobes (formed by the upward convection during intervals of northward IMF) onto plasma fragments when IMF turns southward and the direction of convection velocity changes its sign. The other possible scenario responsible for such short-time PS-plasma structure occurrences was proposed recently by Milovanov et al. (2000). The model extends the earlier one developed for the steady state of the distant magnetotail to the active periods prior to substorm
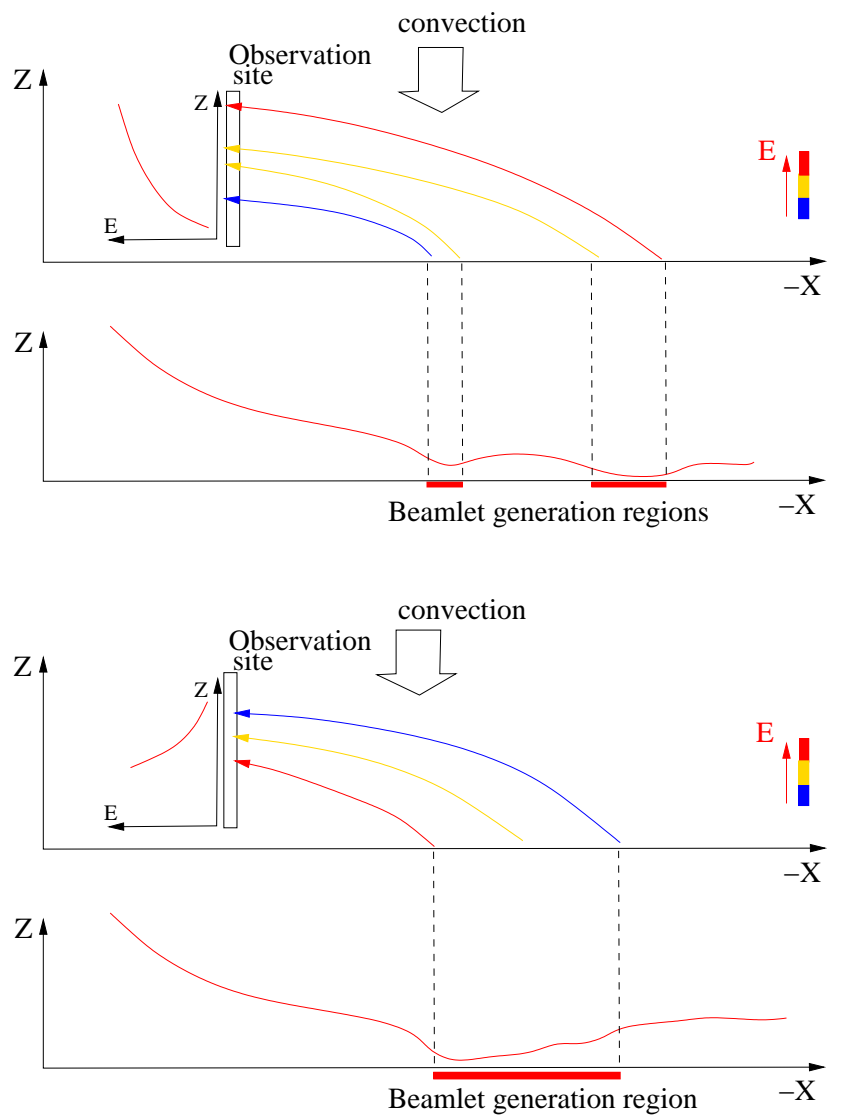

Fig. 13. Two scenario of beamlet energy dispersion. Top panel shows the formation of "normal dispersion" due to both a generally positive $d B_{Z} / d X$ profile and dawn-dusk electric field. The lower panel shows the formation of "inverse dispersion" due to negative $d B_{Z} / d X$. Such a configuration can be the consequence of new near-Earth $X$-line generation.

onset (i.e. when the IMF is predominantly southward). A highly branched network of the cross-tail currents, initially confined to the vicinity of magnetotail field reversal, might become structurally unstable in the course of the energy accumulation in the magnetotail (which should be accompanied by the growth of the average cross-tail current). In this case, some elements of the current network become energetically favourable to bypass the region of the strong magnetic turbulence in the vicinity of the current sheet. At the nonlinear stage of this structural instability, current filaments could emerge from the mid-plane into the regions well outside the PS (see Fig. 15). The sporadic appearance of the current loops in the lobes should be manifested in the observations of the localized regions of PS-like plasma with bulk velocities predominantly directed out of the mid-tail plane. As was argued in Sect. 5, this is just the case for the Interball- 1 observations of PS-like plasma structures, reported in this paper. 

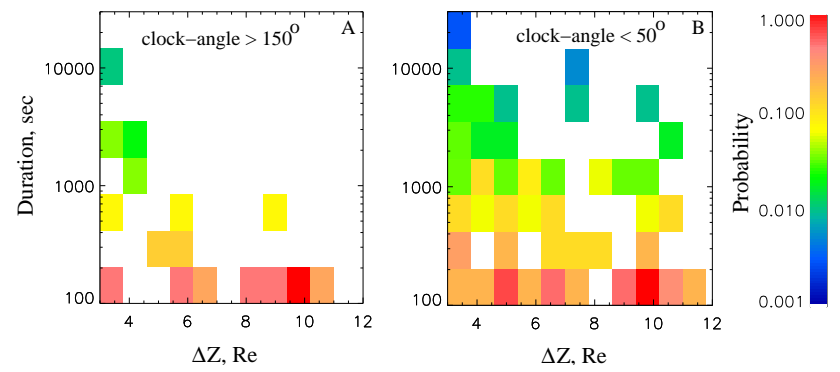

Fig. 14. The duration of PS-like events versus $\Delta Z$. The color-coded histogram shows the probability of observing the event of a particular duration at the particular $\Delta Z$. Panel (a) shows distribution for southward IMF and panel (b) shows the same for northward IMF. Probability is normalized to maximum value.

\section{Summary}

We have studied two types of transient plasma structures observed by Interball- 1 spacecraft in the interface between the magnetotail lobe region and PS: beamlets and quasi-isotropic PS-like plasma structures. Beamlets, non-adiabatically accelerated in the distant localized parts of the magnetotail, are ejected towards the Earth along the outer region of the closed magnetic field lines. Therefore, the occurrence of this structure could serve as an exellent indicator of the boundary between closed and opened magnetic field lines, i.e. the PSBL location. Moreover, as it was shown in the previous section, the dynamics of the beamlet spatial distribution reflects well the magnetospheric conditions and the changes in the reconnection site location in the magnetotail for the various levels of magnetospheric activity.

The distribution of the very small $B_{Z}$ component of the magnetic field in the distant magnetotail (where nonadiabatic beamlet acceleration occurs) strongly controls the ion energy gains. $B_{Z}(X)$-profile, together with $\boldsymbol{E} \times \boldsymbol{B}$ ion velocity filter effect, produces the beamlet energy dispersion in the $Z$-direction observed near the Earth. The form of this dispersion for given magnetospheric and interplanetary conditions allows us to make a suggestion about the character of the $B_{Z}(X)$-profile. We have revealed that the normal energy dispersion and corresponding $B_{Z}$ monotonously decreasing tailward are observed primarily during the quiet periods and when convection in the lobes is directed towards the tail midplane. During the active periods, both normal and inverse beamlet energy dispersions are registered. This fact can be explained by the nonmonotonic profiles of $B_{Z}(X)$ in the region of non-adiabatic beamlet generation. Bursty appearance of beamlets still remains to be explored experimentally (multipoint observations) and theoretically (self-consistent models of non-adiabatic acceleration).

Another indicator of the changes occurring in the magnetotail when IMF turns from the northward to southward direction may be the distribution of the short-time PS-like plasma structures over the tail lobes. During the intervals of the northward IMF, PS-like plasma fills the lobe region
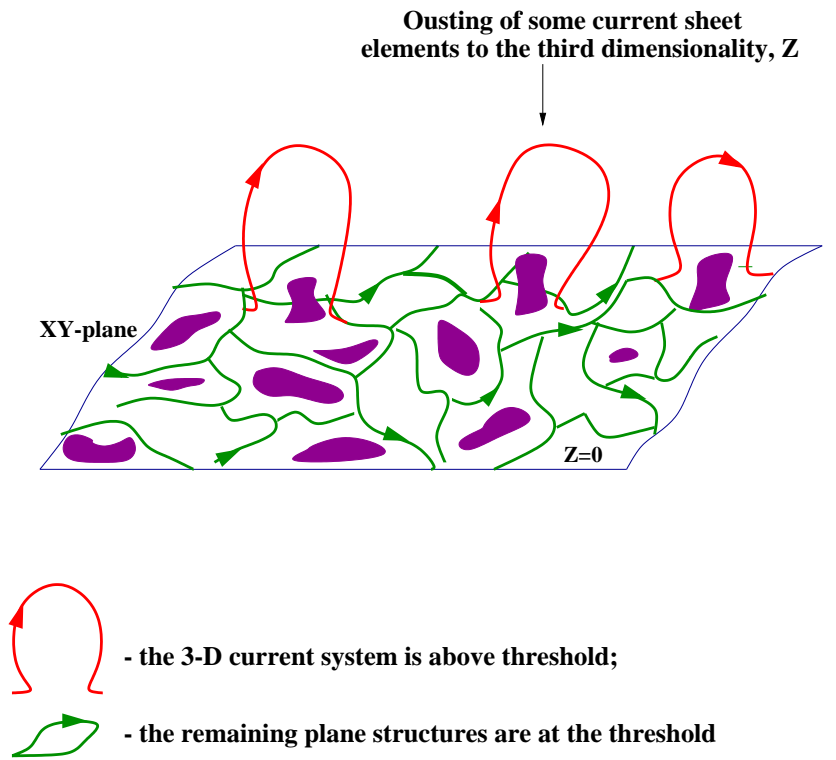

Fig. 15. Scenario of PS filaments formation.

almost entirely and registration of various plasma structures is mainly related to the motions of the PS as the whole entity. But when the IMF turns southward, the observations of plasma structures with long-duration quickly decays with the growth of the distance from NS, while the short-time structures are observed almost uniformly up to the relatively high altitudes over the NS. Registration of such type of structures could hardly be explained by the flapping motions of the PS. It is more probable that the appearance of such structures could be caused by another physical mechanism. It could be the decay of the PS extensions to the lobes (formed by the upward convection during the episodes of the northward IMF) onto plasma fragments (filaments) when the IMF turns southward and the direction of convection changes its sign. As a possible candidate, one could also consider the spatial reconfiguration of the cross-tail current system when average cross-tail current begins to grow before substorm onset. It results in the formation of current loops which are stretched out of the CPS into the lobe region and observed as localised PS-like plasma structures with bulk velocity directed mainly upward.

A further detailed investigation of temporal and spatial features of such plasma structures is required to answer more definitely what are the physical mechanisms responsible for their formation.

Acknowledgement. Authors are grateful to V. Peroomian and M. Ashour-Abdalla for the stimulation discussions of the origin of the beamlets, to L. Avanov, O. Vaisberg and E. Budnik for important comments and help in preparation of the manuscript. This work was supported by the INTAS grants 99-0078 and grants of RFBR NN 00-15-96631 and 01-02-16367.

Topical Editor G. Chanteur thanks two referees for their help in evaluating this paper. 


\section{References}

Ashour-Abdalla, M., Berchem, J. P., Büchner, J., and Zelenyi, L. M.: Shaping of the magnetotail from the mantle: global and local structuring, J. Geophys. Res., 98, 5651-5676, 1993.

Ashour-Abdalla, M., Zelenyi, L. M., Peroomian, V., Richard, R. L., and Bosqued, J. M.: The mosaic structure of plasma bulk flows in the Earth's magnetotail, J. Geophys. Res., 100, 19 191-19209, 1995.

Ashour-Abdalla, M., Frank, L. A., Paterson, W. R., Peroomian, V., and Zelenyi, L. M.: Proton velocity distributions in the magnetotail: theory and observations, J. Geophys. Res., 101, 25872598, 1996.

Baumjohann, W., Paschmann, G., Sckopke, N., Cattel, C. A., and Carlson, C. W.: Average ion moments in the PSBL, J. Geophys. Res., 93, 11 507-11 520, 1988.

Baumjohann, W., Paschmann, G., and Lühr, H.: Characteristics of high-speed ion flows in the plasma sheet, J. Geophys. Res., 95, 3801-3809, 1990.

Belova, E. V., Vaisberg, O. L., Zelenyi, L. M., and Himenes, R. A.: Formation of plasma clouds in high-latitude magnetotail regions, Cosmic Research, 26, 4, 606-619, 1987.

Büchner, J. and Zelenyi L. M.: Deterministic chaos in the dynamics of charged particles near a magnetic field reversal, Physics Letters A, 118, 395-399, 1986.

Büchner, J. and Zelenyi, L. M.: Regular and chaotic charged particle motion in magnetotaillike field reversals 1 . Basic theory of trapped motion, J. Geophys. Res., 94, 11 821-11 842, 1989.

Büchner, J. and Zelenyi, L. M.: The separatrix tentacle effect on the ion acceleration in the PSBL, J. Geophys. Res. Lett., 17, 127130, 1990.

Büchner, J.: Correlation-modulated chaotic scattering in the Earth's magnetotail, Geophys. Res. Lett., 18, 1595-1598, 1991.

Burkhart, G. R. and Chen, J.: Differential memory in the Earth's magnetotail, J. Geophys. Res., 96, 14 033-14 049, 1991.

Chen, J. and Palmadesso, P. J.: Chaos and nonlinear dynamics of single-particle orbits in a magnetotaillike magnetic field, J. Geophys. Res., 91, 1499-1508, 1986.

Chen, J., Burkhardt, G. R., and Huang, C. Y.: Observational signatures of NL magnetotail particle dynamics, J. Geophys. Res. Lett., 17, 2237-2240, 1990.

DeCoster, R. J. and Frank, L. A.: Observations pertaining to the dynamics of the plasma sheet, J. Geophys. Res., 84, 5099-5121, 1979.

Eastman, T. E., Frank, L. A., Peterson, W. K., and Lennartsson, W.: The plasma sheet boundary layer, J. Geophys. Res., 89, 15531572, 1984.

Eastman, T. E., Frank, L. A., and Huang, C. Y.: The boundary layers as the primary transport regions of the Earth's magnetotail, J. Geophys. Res., 90, 9541-9560, 1985.

Eriksson, S., Ergun, R. E., Carlson, C. W., and Peria, W.: The crosspolar potential drop and its correlation to the solar wind, J. Geophys. Res., 105, 18 639-18 654, 2000.

Frank, L. A., Craven, J. D., Gurnett, D. A., Shawhan, S. D., Weimer, D. R., Burch, J. L., Winningham, J. D., Chappell, C. R., Waite, J. H., Heelis, R. A., Maynard, N. C., Sugiura, M., Peterson, W. K., and Shelley, E. G.: The theta aurora, J. Geophys. Res., 91, 3177-3224, 1986.

Frank, L. A., Paterson, W. K., and Kivelson, M.: Observations of nonadiabatic acceleration of ions in Earth's magnetotail, J. Geophys. Res., 99, 14 877-14 890, 1994.

Green, J. L. and Horwitz, J. L.: Destiny of earthward streaming plasma in the plasma sheet boundary layer, Geophys. Res. Lett., 13, 76-79, 1986.

Grigorenko, E. E., Fedorov, A. O., Avanov, L. A., and Zelenyi, L. M.: Transient intermittent structures in the plasma sheet-lobes interface, Proceedings of ICS-5, 493-498, 2000.

Harold, J. B., Chen, J., and Joyce, G.: Resonance-generated particle distributions in magnetotail $X$ line configurations, J. Geophys. Res., 103, $11887-11$ 906, 1998.

Himenez, R. A., Romanov, S. A., Vaisberg, O. L., and Balebanov, V. M.: Plasma flux properties in the magnetotail according to the "Prognoz-8" data, Preprint-930, IKI AN USSR, 1984.

Holland, D. L., Paterson, W. R., Frank, L. A., Kokubun, S., and Yamamoto, Y.: Signatures of nonlinear charged particle dynamics in Geotail comprehensive plasma instrument observations, J. Geophys. Res., 104, 2479-2485, 1999.

Klimov, S., Romanov S., Amata E., Blecki J., and Büchner J., et al.: ASPI experiment: measurements of fields and waves onboard the Interball-1 spacecraft, Ann. Geophysicae, 15, 514-527, 1997.

Lui, A. T. Y., Williams, D. J., Eastman, T. E., and Frank, L. A.: Observation of ion streaming during substorm, J. Geophys. Res., 87, 7753-7764, 1983.

Lyons, L. R. and Speiser, T. W.: Evidence for current-sheet acceleration in the geomagnetic tail, J. Geophys. Res., 87, 2276-2286, 1982.

Milovanov, A., Zelenyi, L. M., and Zimbardo, G.: Topological model for the magnetospheric substorm onset: structural catastrophe of the self-organized critical state, Proceedings of ICS-5, 183-188, 2000.

Moses, R. W., Finn, J. M., and Ling, K. M.: Plasma heating by collisionless magnetic reconnection: analysis and computation, J. Geophys. Res., 98, 4013-4040, 1993.

Ogino, T., Walker, R. J., and Ashour-Abdalla, M.: A global magnetohydrodynamic simulation of the response of the magnetosphere to a northward turning of the interplanetary magnetic field, J. Geophys. Res., 99, 11 027-11 042, 1994.

Onsager T. G., Thomsen, M. F., Gosling, J. T., and Bame, S. J.: Electron distributions in the plasma sheet boundary layer: timeof-flight effects, Geophys. Res. Lett., 17, 1837-1840, 1990.

Onsager, T. G., Thomsen, M. F., Elphig, R. C., and Gosling, J. T.: Models of electron and ion distributions in the plasma sheet boundary layer, J. Geophys. Res., 96, 2099-2101, 1991.

Orsini, S., Candidi, M., and Balsiger, H.: Composition and velocity of ions streaming in the plasma mantle and in the lobe, in: Magnetotail Physics, (Ed) Lui, A. T. Y., 239, John Hopkins University Press, Baltimore, MD, 1987.

Parks, G. K., McCarthy, M., Fitzenreiter, R. J., and Etcheto, J., et al.: Particle and field characteristics of the high-latitude plasma sheet boundary layer, J. Geophys. Res., 89, 8885-8906, 1984.

Parks, G. K., Chen, L. J., McCarthy, M., Larson, D., Lui, R. P., Phan, T., Reme, H., and Sanderson, T.: New observations of ion beams in the plasma sheet boundary layer, Geophys. Res. Lett., 25, 3285-3288, 1998.

Peroomian, V., Ashour-Abdalla, M., and Zelenyi, L. M.: Intrinsic Variability in the quiet-time magnetotail, in: Magnetospheric Current Systems, Geophysical Monograph 118, (Ed) the American Geophysical Union, 305-312, 2000.

Sitnov, M. I., Zelenyi, L. M., Malova, H. V., and Sharma, A. S.: Thin current sheet embedded within a thicker plasma sheet: selfconsistent kinetic theory, J. Geophys. Res., 105, 13 029-13 043, 2000 .

Takahashi, K. and Hones, E. W.: ISEE 1 and 2 observations of ion distributions at the plasma sheet-tail lobe boundary, J. Geophys. 
Res., 93, 8558-8582, 1988.

Walker R. J., Richard R. L., Ogino, T., and Ashour-Abdalla, M.: The response of the magnetotail to changes in the IMF orientation: The magnetotail's long memory, Physics and Chemistry of the Earth, 24, 221-227, 1999.

Winningham, J. D., Yasuhara, F., Akasofu, S.-I., and Heikkila, W. J.: The latitudinal morphology of $10-\mathrm{eV}$ to $10-\mathrm{keV}$ electron fluxes during magnetically quiet and disturbed times in the 21:00-03:00 MLT sector, J. Geophys. Res., 80, 3148-3171, 1975.

Wygant, J. R., Torbert, R. B., and Mozer, F. S.: Comparison of S3-3 polar cap potential drops with the interplanetary magnetic field and models of magnetopause reconnection, J. Geophys. Res., 88, 5727-5735, 1983.

Yermolaev, Yu. I., Fedorov, A. O., Vaisberg, O. L., Balebanov, V. M., and Obod, Y. A., et al.: Ion distribution dynamics near the Earth'sbow shock: first measurements with the 2D ion energy spectrometer CORALL on the Interball-Tail satellite, Ann. Geophysicae, 15, 533-541, 1997.

Zelenyi L. M., Delcourt, D., Malova, H. V., and Sharma, A. S., et al.: Forced current sheets in the Earth's magnetotail: its role and evolution due to nonadiabatic particle scattering, in: Proc. of COSPAR colloquia, Advances Space Res., in press, 2001.

Zelenyi, L. M., Kovrazkhin, R. A., and Bosqued, J. M.: Velocitydispersed ion beams in the nightside auroral zone: AUREOL-3 observations, J. Geophys. Res., 95, 12 119-12 139, 1990.

Zelenyi, L. M., Taktakishvili, A. L., Dubinin, E. M., Budnik, E. Yu., and Sandahl, I.: Ion beam velocity distributions in plasma sheet boundary layer, Proceedings of the 9th COSPAR Colloquium "Magnetospheric Research with Advanced Techniques", Beijing, China, 125-132, 1996.

Zelenyi, L. M., Sitnov, M. I., Malova, H. V., and Sharma, A. S.: Thin and superthin ion current sheets. Quasi-adiabatic and nonadiabatic models, Non. Proc. Geophys., 7, 127-139, 2000. 